\title{
An Iterative Method for the Uniformity Improvement of Edge-Lit Backlight
}

\author{
Jin-Ren Yan, De-Cong Li, Zhi-Kun Wang, and Wen-Ping Rao \\ Department of Optoelectronic Engineering, Yunnan Open University, Xuefu Road 113, Kunming 650223, China \\ Correspondence should be addressed to Jin-Ren Yan; renyj0720@163.com
}

Received 16 June 2016; Accepted 5 September 2016

Academic Editor: Yuqin Zong

Copyright (C) 2016 Jin-Ren Yan et al. This is an open access article distributed under the Creative Commons Attribution License, which permits unrestricted use, distribution, and reproduction in any medium, provided the original work is properly cited.

\begin{abstract}
A simple iterative optimization method is developed to improve the exiting light uniformity of the edge-lit backlight module. The method uses the relative deviation of the average luminous flux of the entire light guide plate (LGP) and the luminous flux of each region as constraint criterion and the ratio of the luminous flux of each region to the average luminous flux of the LGP as adjustment coefficient to adjust the density of the microstructures in each region. This process loops until the relative deviations of all regions meet a prespecified threshold value. A LGP based on microprism structure is presented to validate the method. The initial values, which represent the size of the microstructures manufactured on the bottom surfaces of the LGP, $d_{0}$-dependent luminous fluxes, microstructure size distributions, iterations of the LGP, and the relative deviation of each region are discussed. The simulation results show that it is a very practical method for the uniformity improvement of the backlight when an appropriate initial value is selected and the final uniformity is better than $90 \%$ after optimization.
\end{abstract}

\section{Introduction}

Liquid crystal display (LCD) is widely used in our life for its advantages of low cost, low power consumption, no radiation, lightweight, and high video quality. However, the LCD panel is not a self-emissive display device; a uniform illumination backlight is needed to illuminate the LCD panel for image display. Particularly for small and medium sized LCD panels, a high performance light guide plate (LGP) is the most important component of the LCD backlight module. In the past few years, researchers have made efforts to propose new structures of LGPs to increase the efficiency of the LCD backlight module [1-4] or develop useful methods to improve the uniformity of the backlight. Lee et al. [5] first used experiments to design the incoupling part of LGP and then a density based approach with progressive quadratic response surface modeling was used to optimize the dot patterns; the uniformity reached $82 \%$. This method requires a special software package for microstructure optimization. Li et al. [6] designed a prism-pattern LGP "in a way" first, and then a curve fitting method was used to further improve the uniformity of the backlight and the illuminance uniformity is higher than $90 \%$ by using this method. However, before further optimization, designers need to perform a large number of optical simulations to get a better distribution of microstructures using this method. Xu et al. [7] introduced a neural-network optical model and a genetic algorithm to achieve excellent luminance uniformity in the exiting light. However, it requires a lot of repeated optical simulation to construct the neural-network optical model of LGP. Kusko et al. [8] proposed an analytical approach for LGP optimization. Using this method, designers need to perform complex mathematical derivations. Moreover, the authors only designed a LGP with a width of $0.1 \mathrm{~mm}$; it is unpractical in real application. Chang and Lee [9] proposed a dot-pattern generation scheme by using a molecular dynamics method. However, the dot patterns generated by this method are randomly distributed on the bottom surface of the LGP; such dot patterns are difficult to manufacture. Zhao et al. [10] developed a fuzzy scheme strategy to generate dot patterns for light guide plate. However, this method includes fuzzification and defuzzification strategies, and it is somewhat complex when compared with other methods. 


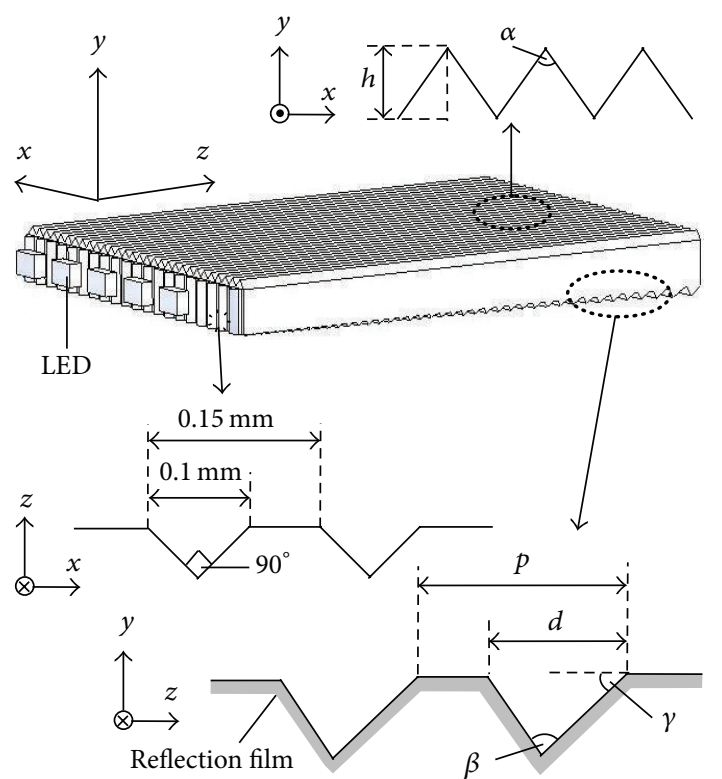

Figure 1: The microprism LGP used to demonstrate the iterative method.

Different from the previous works, in this study, we introduce an iterative method for the uniformity improvement of the edge-lit backlight module. The method presented in this work only employs the relative deviation of the average luminous flux of the entire LGP and the luminous flux of each region as constraint criterion and the ratio of the luminous flux of each region to the average luminous flux of the LGP as adjustment coefficient to adjust the density of the microstructures in each region. After a proper initial value of the microstructures across the entire LGP is selected, the method can automatically search for the optimal microstructure distribution on the bottom surface of LGP. Compared with other methods, it is simple and does not need any complex mathematical calculations or a large number of repetitive trial-and-error processes.

\section{Structure of the LGP}

Figure 1 shows the structure of an integrated LGP based on microprism structures $[11,12]$. The light source consists of several LEDs that have been arranged in a line along the direction of the $x$-axis. The front, top, and bottom surfaces of the LGP are microprism structures. The directions of the microprisms on the front, top, and bottom surfaces are, respectively, designed along the directions of the $y$-, $z$-, and $x$-axes of the coordinate system. The microprisms on the front and top surfaces are designed in constant size and the size of the microprisms on the bottom surface is monotonically increasing along the direction of the $z$-axis. The bottom of the LGP is coated with high reflective material which causes specular reflection; the three side faces contain reflection sheets which are used to reflect lights back into LGP and the light emitted from the top surface of the LGP. $h$ and $\alpha$ represent the height and apex angle of the microprisms on the top of the LGP, respectively. $p, \beta$, and $d$ are the period, apex

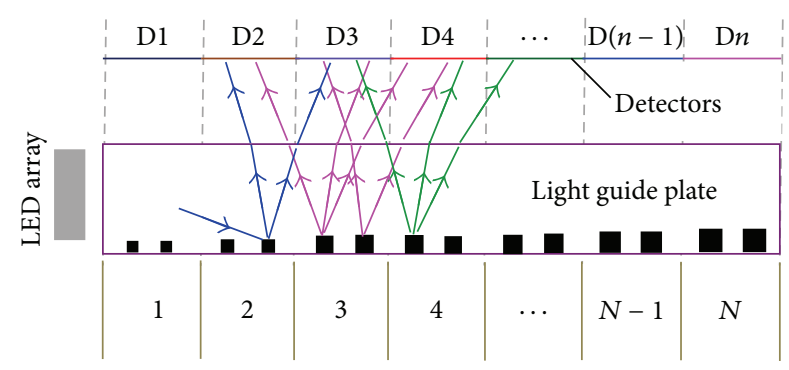

FIGURE 2: The diagram of the regional partition method for the LGP and the mechanism of the light rays redirected by microstructures.

angle, and width of the microprism on the bottom of the LGP, respectively.

\section{The Iterative Method}

Based on the design principle of LGP, to achieve uniform illumination at the light exiting surface of LGP, the density of the microstructures on the bottom surface of LGP should increase gradually with the increase of distance from the light source. However, it is difficult to directly carry out a mathematical expression for the optimal microstructure distribution on the bottom surface of LGP by mathematical derivation method or analytical method because the microstructure distribution is usually a nonlinear function for a uniform exiting light. A widely used method for LGP uniformity optimization is regional partition method; the method first divides the microstructures on the bottom surface of LGP into several independent regions and then uses proper optimization method to adjust the density of microstructures in each region until uniform illumination is obtained $[5-7,9,10]$.

According to the contents described above, in this study, the LGP is first divided into $N$ equal regions along the light propagating direction and the density of the microstructures in each region will be optimized, as shown in Figure 2. Detectors of these regions are placed above to collect light rays. Then, we only need to search for a set of density combinations of the microstructures in these regions to make each region have equal light energy. As long as a sufficient number of regions are divided, high uniform illumination can be obtained at the light exiting surface of the LGP. Figure 2 also shows the possible directions when the total internal reflection condition of the light rays is damaged by microstructures in each region. As illustrated in the figure, the redirected light of a region may fall into the adjacent region. This means that when the microstructure's density of a region changes, not only the luminous flux of this region, but also the luminous fluxes of the adjacent regions will change. So it is difficult to obtain a uniform exiting light by adjusting the density of the microstructures in each region separately. In this study, we will show an iterative method used to address this difficulty. The core idea of this method is that the densities of the microstructures of all regions are iteratively adjusted at the same time until a set of optimal distributions is reached, namely, uniform illumination obtained at the light exiting surface. Detailed design procedure of the method is described 
in the following. Note that the density of the microstructures is adjusted by adjusting the size of the microstructures in each region.

By using optical simulation software, we could obtain the total luminous flux $\Phi_{\text {total }}$ of the entire LGP and the luminous flux $\Phi_{i}(i=1,2, \ldots, N)$ of each region. Then, the average luminous flux of the entire LGP can be calculated by

$$
\Phi_{\mathrm{ave}}=\frac{\Phi_{\mathrm{total}}}{N}
$$

where $\Phi_{\text {ave }}$ and $N$ represent the average luminous flux of the LGP and the number of regions, respectively. The definition of the relative deviation of the luminous flux and the adjustment coefficient are expressed as

$$
\begin{aligned}
& \delta_{i j}=\left|\frac{\Phi_{i j}-\Phi_{\mathrm{ave}, j}}{\Phi_{\mathrm{ave}, j}}\right| \times 100 \%, \\
& k_{i j}=\frac{\Phi_{i j}}{\Phi_{\mathrm{ave}, j}},
\end{aligned}
$$

where $\delta_{i j}$ and $k_{i j}$ represent the relative deviation of the luminous flux and the adjustment coefficient which will be served as constraint criterion and adjustment coefficient to optimize the LGP, respectively. Note that $i$ and $j$ represent the region number and the number of iterations, respectively. So $\Phi_{i j}, \delta_{i j}$, and $k_{i j}$ represent the luminous flux, relative deviation, and adjustment coefficient of the $i$ th region in the $j$ th iteration, respectively, and $\Phi_{\text {ave, } j}$ represents the average luminous flux of the entire LGP in the jth iteration.

For uniform illumination, the luminous flux of each region $\Phi_{i j}$ should approximate the average luminous flux

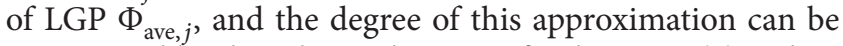
represented by the relative deviation $\delta_{i j}$ shown in (2). When the relative deviation $\delta_{i j}$ of a region is greater than a small preset value, such as $1 \%$ used in this study, the microstructure size $d_{i j}$ of this region will be adjusted by

$$
d_{i, j+1}=\frac{d_{i j}}{k_{i j}} .
$$

Note that (4) implies the following principle: when a region contains higher luminous flux than the average luminous flux of the LGP (i.e., $k_{i j}>1$ ), the size of the microstructures in this region should be adjusted to a smaller value. In other words, a region contains lower luminous flux than the average luminous flux of the LGP (i.e., $k_{i j}<1$ ), and the microstructure size of this region should be increased.

After the size of the microstructures in each region is calculated by (4), the simulation and computation will go into the next iteration. Then, the above operation repeats until the relative deviations of all regions meet the requirement (i.e., $\delta_{i j} \leq 1 \%$ for all regions). It is worth noting that since all regions of the LGP are optimized simultaneously, when the relative deviation $\delta_{i j}$ of a region is less than or equal to the threshold value in an iteration, the adjustment coefficient of this region is set as $k_{i j}=1$ for the purpose of keeping the density of the microstructures in this region unchanged

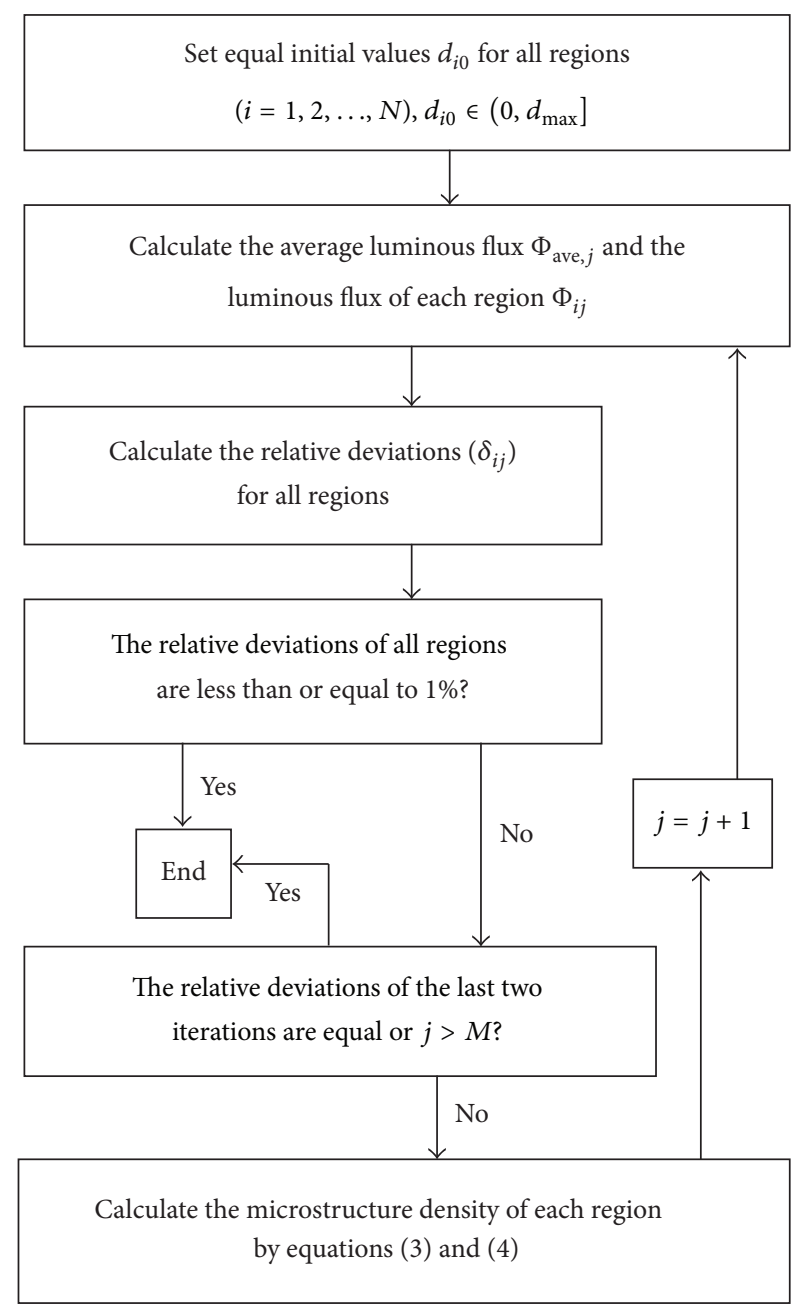

Figure 3: The flow chart of the iterative method.

in the next iteration. According to the contents described above, the design flow chart of the iterative method is shown in Figure 3. As shown in Figure 3, the iterative process will terminate in case the following two conditions are met: (1) the relative deviations of the last two iterations are equal and (2) the number of iterations reaches the maximum value (i.e., the number $M$ shown in the flow chart).

\section{Simulations and Discussions}

Based on the design procedure described above, a LGP based on microprism structure is optimized to demonstrate the effectiveness of the iterative method. It is a rectangular shape with 2.5-inch size, and the length, width, and thickness of the LGP are $60 \mathrm{~mm}, 40 \mathrm{~mm}$, and $3 \mathrm{~mm}$, respectively. It is made of PMMA with a refractive index of $n=1.49$ and the reflectivity of the reflective material is $95 \%$. The LGP is divided into 12 equal regions. In order to analyze the performance of this method, the initial value range of the microprism on the bottom surface of LGP is set as $d_{i 0}=(0.05-0.1) \mathrm{mm}$. The maximum value of the microprism is $d_{\max }=0.2 \mathrm{~mm} . \beta, \gamma$, $\alpha, h$, and $p$ of the microprism structure shown in Figure 1 
TABLE 1: Initial values $d_{0}$-dependent iterations.

\begin{tabular}{cccccccc}
\hline & \multicolumn{7}{c}{ Microprism } \\
& 0.05 & 0.06 & 0.07 & 0.075 & 0.08 & 0.09 & 0.10 \\
\hline Iterations & 5 & 6 & 7 & 6 & 8 & 14 & 17 \\
\hline
\end{tabular}

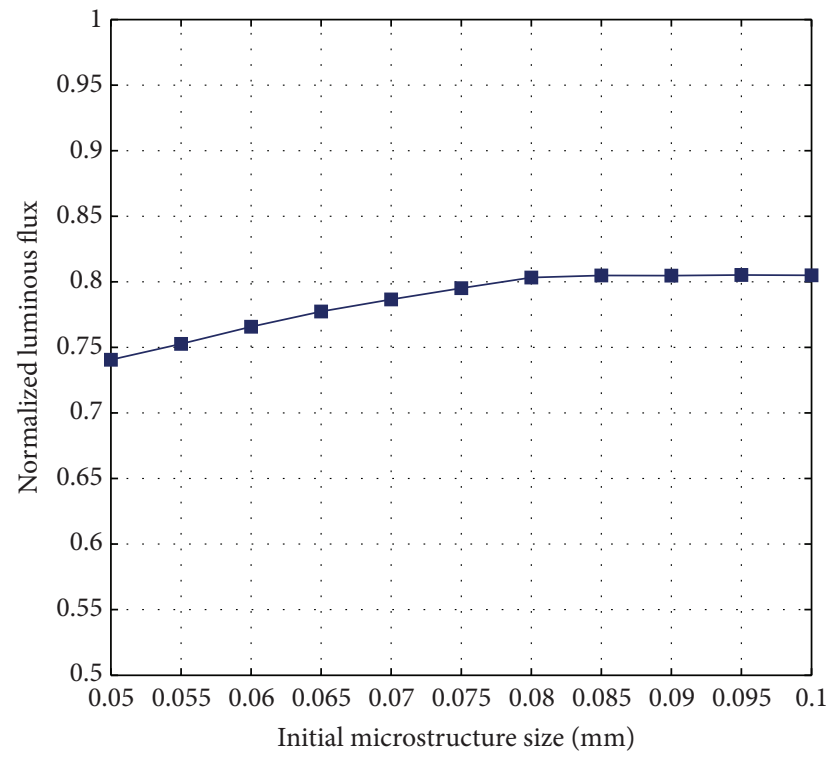

FIGURE 4: Initial values $d_{0}$-dependent luminous flux of the LGP.

are $45^{\circ}, 35^{\circ}, 100^{\circ}, 0.04 \mathrm{~mm}$, and $0.2 \mathrm{~mm}$, respectively [12]. The maximum number of iterations is 60 (i.e., $M=60$ ). The total luminous flux of the light sources is $100 \mathrm{~lm}$, and 1.3 million rays were used for simulation.

The initial values $d_{0}$-dependent luminous flux of the LGP is shown in Figure 4. One can note that the luminous flux of the LGP monotonically increases with $d_{0}$ at first, and then it almost keeps constant after reaching a certain value. The reason can be explained by Figure 5. From the figure, one can note that the microstructure distribution curve along with the distance from the light source obtains a small increment with a small initial value; therefore, a portion of light rays cannot couple out from LGP effectively. When one sets initial values larger than $0.075 \mathrm{~mm}$, the size distribution curves were almost the same, so the corresponding luminous fluxes of the LGP almost keep constant.

The initial values $d_{0}$-dependent relative deviations of the microprism structure LGP are shown in Figure 6. One can note that when we set initial value of the microprisms greater than or equal to $0.08 \mathrm{~mm}$, the relative deviations of the regions far away from the light source are larger than the preset threshold value. This means that when a large initial value of microprisms is selected, the luminous flux values of the regions far away from the light source will be too small.

Table 1 shows the corresponding iterations for different initial values. From the results shown in the table, one can conclude that the developed iterative method only needs several iterations for the LGP based on microprism structure.

According to the discussions above, we note that $0.075 \mathrm{~mm}$ is an optimal initial value for a better result. Thus,

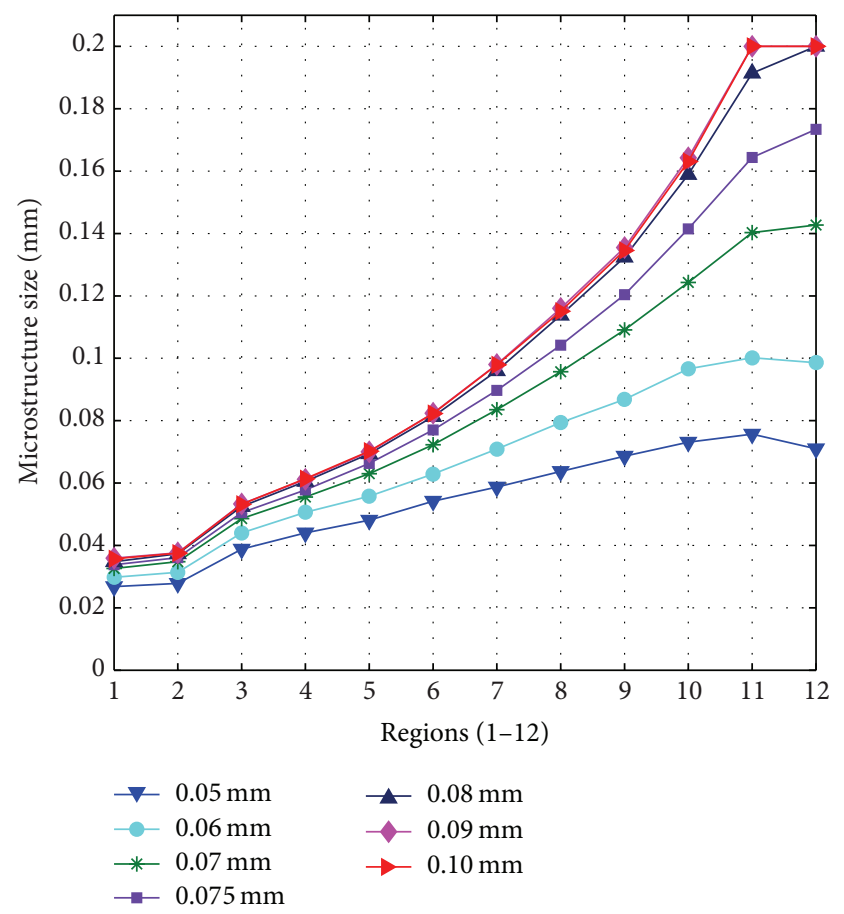

FIgURE 5: Initial values $d_{0}$-dependent microstructure size distribution curves.

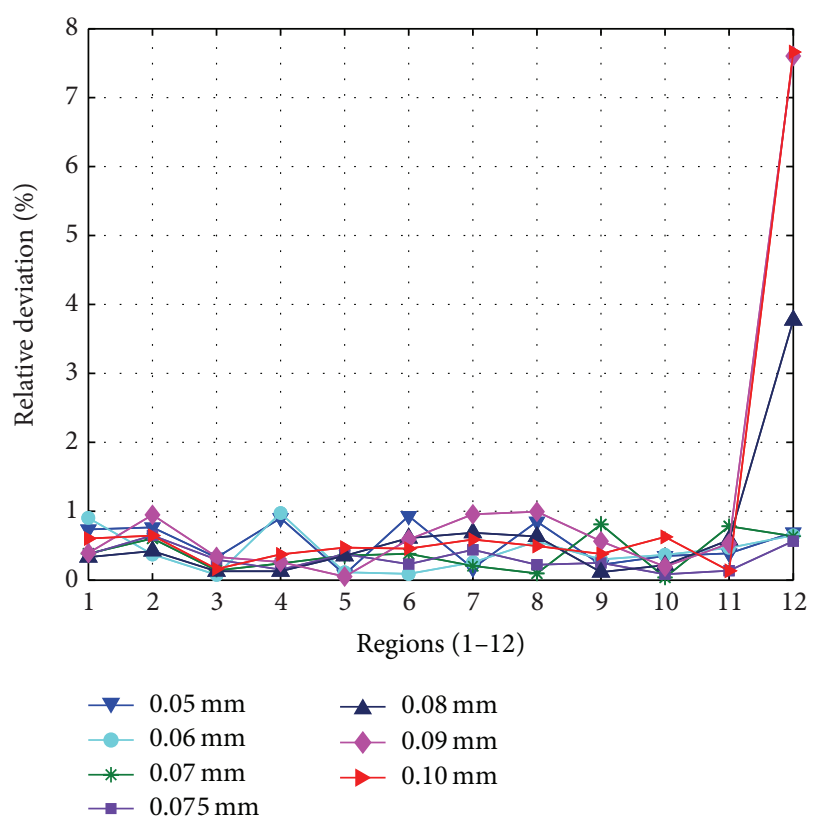

FIGURE 6: Initial values $d_{0}$-dependent relative deviations.

the nine-point method is used to evaluate the uniformity of the edge-lit backlight module when the initial value of the microprisms is $0.075 \mathrm{~mm}$. The uniformity is defined as the ratio of the minimum luminance to the maximum luminance and is expressed as

$$
U=\frac{L_{\min }}{L_{\max }} \times 100 \%
$$




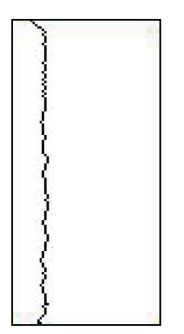

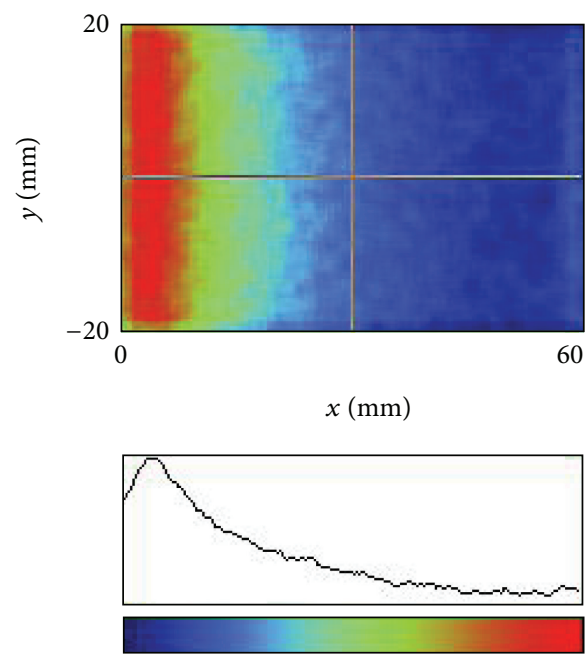

(a) Before optimization
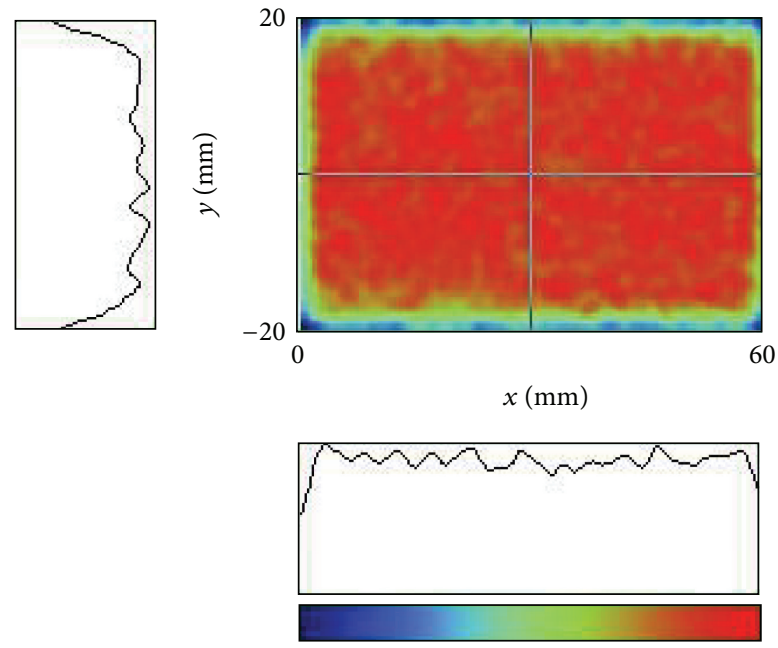

(b) After optimization

FIGURE 7: Irradiance maps before and after optimization of the microprism LGP.

where $L_{\min }$ and $L_{\max }$ represent the minimum luminance and maximum luminance, respectively, and $U$ represents the uniformity of the backlight. Figure 7 shows the irradiance maps of the backlight before and after optimization when the initial value is $0.075 \mathrm{~mm}$ and the uniformity improves from $39.87 \%$ to $91.30 \%$.

\section{Conclusion}

In this study, we developed a simple iterative optimization method to improve the illumination uniformity of the edgelit backlight module. A 2.5-inch LGP based on microprism structure was constructed to demonstrate the feasibility of the method and the calculation principle of the iterative method was described in detail. The dependencies of the luminous fluxes, microstructure density distributions, and the relative deviations on the initial values were discussed. The result showed that the final luminance uniformity of the backlight is better than $90 \%$ with a proper initial value.

\section{Competing Interests}

The authors declare that they have no competing interests.

\section{Acknowledgments}

This work is supported by scientific research fund of Yunnan Open University under Grant no. 2014Y011 and by Yunnan Province Department of Education Fund under Grant no. $2014 Z 143$.

\section{References}

[1] J.-W. Pan and Y.-W. Hu, "Design of a hybrid light guiding plate with high luminance for backlight system application," Journal of Display Technology, vol. 9, no. 12, pp. 965-971, 2013.

[2] P.-H. Yao, C.-J. Chung, C.-L. Wu, and C.-H. Chen, "Polarized backlight with constrained angular divergence for enhancement of light extraction efficiency from wire grid polarizer," Optics Express, vol. 20, no. 5, pp. 4819-4829, 2012.
[3] Z. Luo, Y.-W. Cheng, and S.-T. Wu, "Polarization-preserving light guide plate for a linearly polarized backlight," IEEE/OSA Journal of Display Technology, vol. 10, no. 3, pp. 208-214, 2014.

[4] C.-F. Chen and S.-H. Kuo, "A highly directional light guide plate based on V-groove microstructure cell," Journal of Display Technology, vol. 10, no. 12, pp. 1030-1035, 2014.

[5] G. Lee, J. H. Jeong, S.-J. Yoon, and D.-H. Choi, "Design optimization for optical patterns in a light-guide panel to improve illuminance and uniformity of the liquid-crystal display," Optical Engineering, vol. 48, no. 2, Article ID 024001, 2009.

[6] C.-J. Li, Y.-C. Fang, W.-T. Chu, and M.-C. Cheng, "Optimization of light guide plate with microstructures for extra light modern backlight module," Japanese Journal of Applied Physics, vol. 47, no. 8, pp. 6683-6687, 2008.

[7] P. Xu, Y. Huang, Z. Su, and X. Zhang, "Algorithm research on microstructure distribution on the bottom surface of an integrated micro-optical light guide plate," Applied Optics, no. 7, pp. 1322-1327, 2014.

[8] M. Kusko, C. Kusko, and D. Cristea, "Method of determination of light-scatterer distribution in edge-lit backlight units using an analytical approach," Journal of the Optical Society of America A, vol. 27, no. 9, pp. 2015-2020, 2010.

[9] J.-G. Chang and C.-T. Lee, "Random-dot pattern design of a light guide in an edge-lit backlight: integration of optical design and dot generation scheme by the molecular-dynamics method," Journal of the Optical Society of America A, vol. 24, no. 3, pp. 839-849, 2007.

[10] H. Zhao, S. P. Fang, Q. Yang, and B. Shang, "Generation of diffuser dot patterns for light guides using fuzzy scheme strategy," Optical Engineering, vol. 48, no. 12, Article ID 124001, 2009.

[11] D. Feng, G. Jin, Y. Yan, and S. Fan, "High quality light guide plates that can control the illumination angle based on microprism structures," Applied Physics Letters, vol. 85, no. 24, pp. 6016-6018, 2004.

[12] J.-R. Yan, Q.-H. Wang, D.-H. Li, and J.-D. Zhang, "Edgelighting light guide plate based on micro-prism for liquid crystal display," Journal of Display Technology, vol. 5, no. 9, pp. 355-357, 2009. 


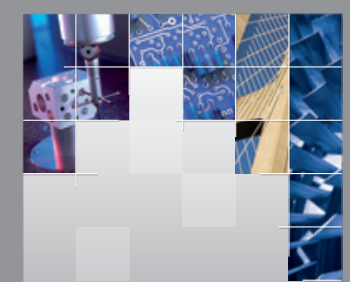

\section{Enfincering}
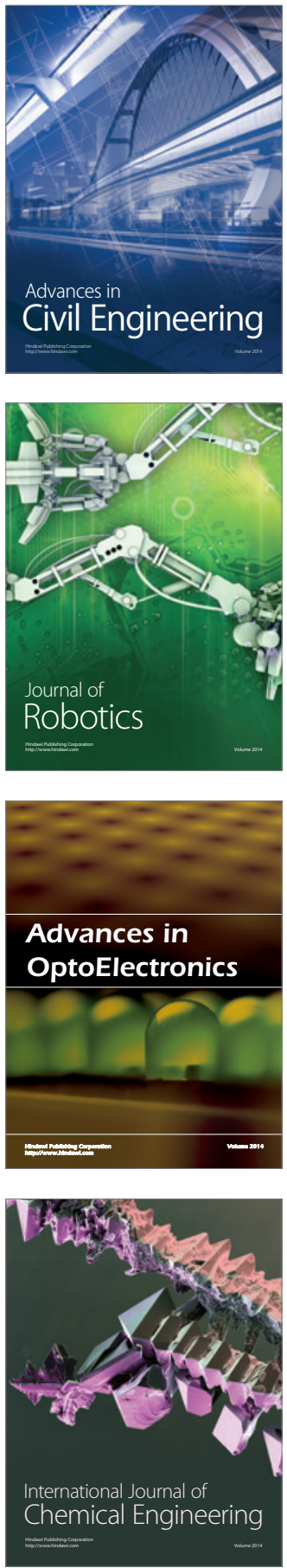

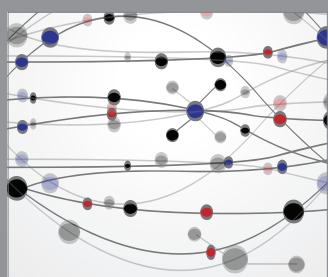

The Scientific World Journal

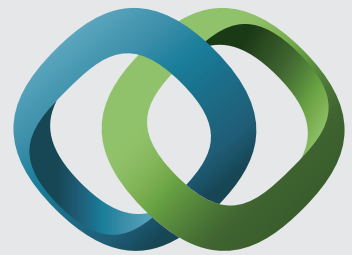

\section{Hindawi}

Submit your manuscripts at

http://www.hindawi.com
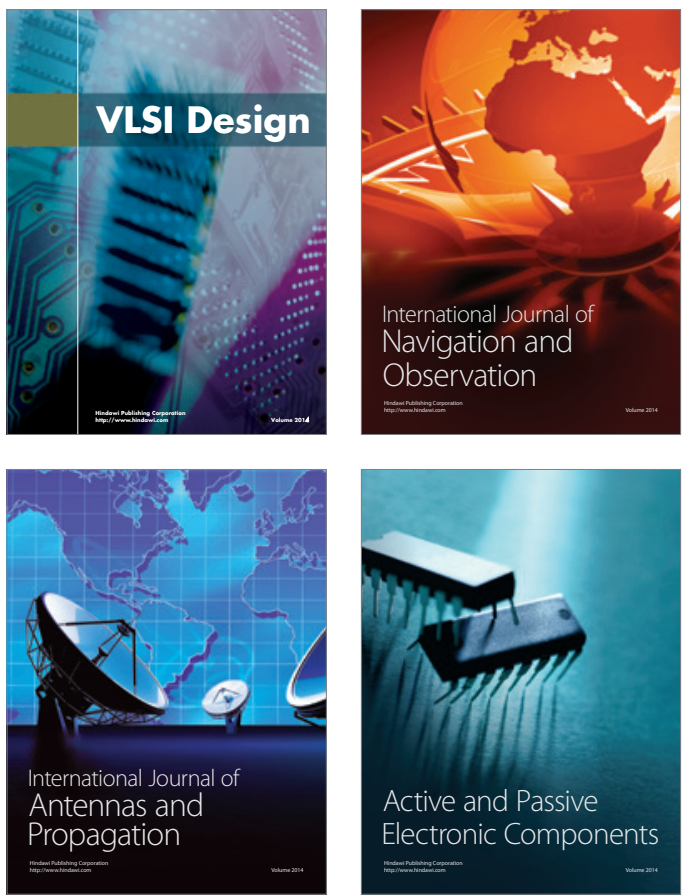
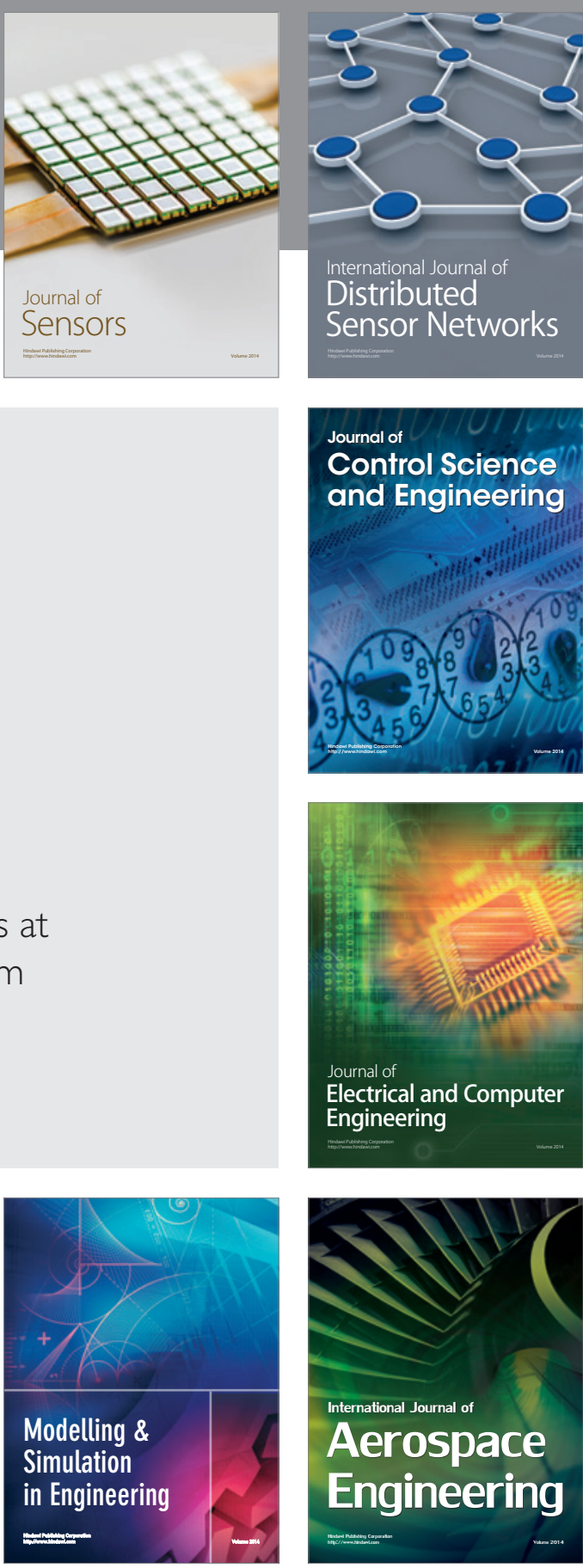

International Journal of

Distributed

Sensor Networks

Journal of

Control Science

and Engineering
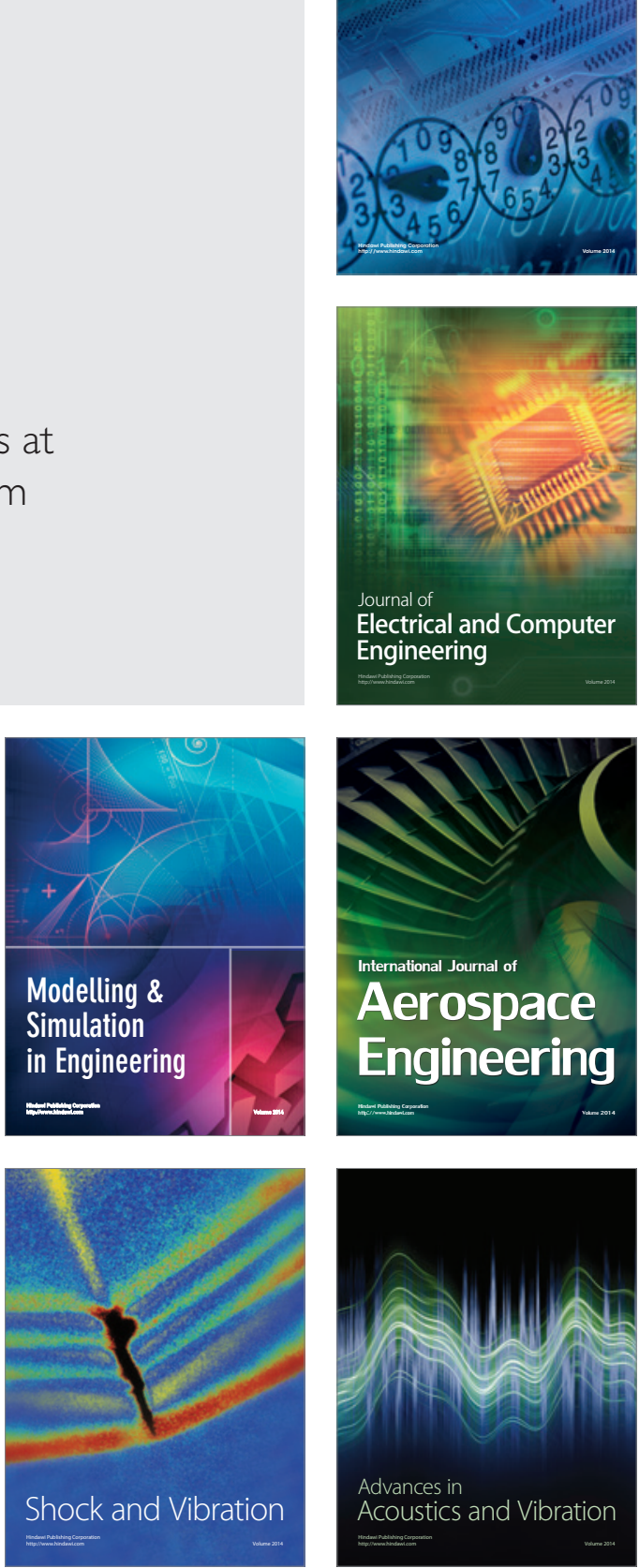